\title{
Comments on Statistical Issues in January 2016
}

\author{
Kyung Do Han, Yong Gyu Park \\ Department of Biostatistics, The Catholic University of Korea College of Medicine, Seoul, Korea
}

In this section, we would like to discuss the purpose of propensity score matching in analyzing Korea National Health and Nutrition Examination Survey (KNHANES) data, which is presented in the article titled, "The association between atopic dermatitis and depressive symptoms in Korean adults: The fifth Korea National Health and Nutrition Examination survey, 2007-2012," published in November 2015 by Kim et al. ${ }^{1)}$

\section{PURPOSE OF MATCHING IN STATISTICAL ANALYSES}

The most problematic aspect in retrospective studies or cross-sectional studies is whether the homogeneity is maintained between the comparative groups. When there is a possibility that the factors involved affect the therapeutic effect as much as the treatments themselves and have same distributions in both treatment groups, it is enough to compare the difference between the treatment groups directly without adjusting those factors to compare the therapeutic effects of two treatments. However, if those factors are distributed differently in the groups, also known as confounding variables, it is difficult to determine if the difference in the therapeutic effect is a consequence of the treatment or the confounding variables. In this study, we (1) performed multivariate (multiple) analyses such as a multiple regression, analysis of covariance, and a multiple logistic regression to adjust their influence over the result, or (2) pair upin other words, match up-subjects from each group with same or similar factor values prior to the analysis.

In general, matched data are not considered independent. Thus, statistical analysis methods to compare dependent groups should be used, including paired t- test, McNemar test, and conditional logistic regression. On the other hand, if the matching is for the purpose of satisfying the inclusion or exclusion criteria for the subjects, it is not necessary to use the statistical methods for paired data. Kim et al. ${ }^{1)}$ matched the subjects based on sex and age using propensity score matching, ${ }^{2)}$ regarded them as independent groups, and used the appropriate statistical methods for independent data.

\section{1:5 MATCHING}

Many researchers find it difficult to apply weights when analyzing the KNHANES data. KNHANES data are obtained by a complex, stratified, and multistage cluster sampling design. Therefore, it is important to analyze the data using proper weights provided herewith. 'Proper weights' refers to each observation in KNHANES data that is obtained by different sampling probability. On the other hand, most well known statistical methods assume that each observation is based on a simple random sampling; in this study, all observations have similar sampling probabilities (weights).

Kim et al. ${ }^{1)}$ analyzed $434 \mathrm{AD}$ patients and 2,170 ageand sex-matched control subjects via 1:5 propensity score matching. However, as mentioned above, if we consider each observation has a different weight, the ' $1: 5$ matching' is not a precise expression.

\section{CONFLICT OF INTEREST}

No potential conflict of interest relevant to this article was reported. 


\section{REFERENCES}

1. Kim S, Lee JY, Oh JY, Chekal L, Lee DC. The association between atopic dermatitis and depressive symptoms in Korean adults: the fifth Korea
National Health and Nutrition Examination Survey, 2007-2012. Korean J Fam Med 2015;36:261-5.

2. Park YG. Comments on statistical issues in February. Korean J Fam Med 2011;32:144-5. 